\title{
Treatment with cyclosporin switching to hydroxychloroquine in patients with rheumatoid arthritis
}

\author{
W-U Kim, Y-I Seo, S-H Park, W-K Lee, S-K Lee, S-I Paek, C-S Cho, H-H Song,
} H-Y Kim

Centre for Rheumatic Disease, Kang-Nam St Mary's Hospital, School of Medicine, The Catholic University of Korea, Seoul, Republic of Korea

W-U Kim

Y-I Seo

S-H Park

S-I Paek

C-S Cho

H-Y Kim

Department of Rheumatology, Yonsei University College of Medicine, Seoul, Republic of Korea W-K Lee

S-K Lee

Department of Biostatistics, The Catholic University of Korea, Seoul, Republic of Korea

$\mathrm{H}-\mathrm{H}$ Song

Correspondence to: Dr H-Y Kim, Division of Rheumatology, Department of Internal Medicine, School of Medicine, The Catholic University of Korea, Centre for Rheumatic Diseases in Kang-Nam St Mary's

Hospital, 505 Banpo-Dong, Seocho-Ku, Seoul, 137-040, Korea

rheuma@cmc.cuk.ac.kr

Accepted 3 October 2000

\begin{abstract}
Objective-To investigate the therapeutic benefit of cyclosporin A (CSA) switching to hydroxychloroquine (HCQ) in patients with rheumatoid arthritis (RA).

Methods-Thirty four patients with RA who displayed residual inflammation and disability despite partial responses to prior maximal tolerated doses of methotrexate, were included. All were treated with a staged approach using CSA for 24 weeks to induce clinical improvement, followed by HCQ for 16 weeks to maintain the improvement. Seven ACR core set measures were evaluated every four to eight weeks.
\end{abstract}

Results-During a 40 week open trial, 27/34 patients completed the study. CSA treatment significantly reduced the tender joints score, swollen joints score, visual analogue pain scale, patient's or doctor's global assessment, patient's self assessed disability, and $\mathrm{C}$ reactive protein. Compared with the time of entry into the trial, patients who switched from CSA to HCQ still possessed significantly lower levels of most variables, determined at 28,32 , and 40 weeks. According to the ACR 20\% improvement definition, 15/27 (56\%) patients had improved at 24 weeks after CSA treatment, and $14 / 27(52 \%)$ remained improved at 16 weeks after the change to HCQ. Frequent side effects, such as hypertrichosis, gastrointestinal trouble, and hypertension, were noted during CSA treatment, but most of these disappeared after switching to HCQ. The mean levels of blood pressure and serum creatinine were significantly increased during CSA treatment, but returned to normal after changing to HCQ.

Conclusions-The data suggest that CSA switching to HCQ treatment may be an effective strategy for patients with RA partially responding to methotrexate, particularly those with toxicity due to CSA. (Ann Rheum Dis 2001;60:514-517)

In the treatment of rheumatoid arthritis (RA), there is a large group of patients who do not respond to single disease modifying antirheumatic drugs (DMARDs). Triple treatment with hydroxychloroquine (HCQ), sulfasalazine, and methotrexate (MTX) can be given to these patients because its effect may be substantially better than MTX alone. ${ }^{1}$ Recent trials have suggested that cyclosporin A (CSA) is also effective in the treatment of patients with RA who partially respond to single DMARDs. ${ }^{2-4}$ However, the major problem with CSA is its side effects, such as renal toxicity, hypertension, and gastrointestinal complaints, which may limit its use.

In one trial of the effects of low dose CSA compared with chloroquine in RA, it was shown that chloroquine may be as effective as $\mathrm{CSA},{ }^{5}$ though some studies conclude that HCQ has low efficacy. ${ }^{6}$ Moreover, HCQ showed the least toxicity of several DMARDs. ${ }^{67}$ Consequently, HCQ seems to be a good candidate for the replacement of CSA in patients treated with MTX and CSA. This study aimed at investigating the therapeutic benefit of CSA switching to HCQ in patients with RA.

\section{Patients and methods}

PATIENTS

Patients who fulfilled the revised criteria of the American Rheumatism Association (ARA) for RA, ${ }^{8}$ aged 20-59, and who showed a partial response to MTX, were selected from among patients who were monitored from June 1997 to December 1998 in two medical centres, Kang-Nam St Mary's Hospital and Severance Hospital. The patients partially responding to MTX were patients who had active synovitis, defined as six or more actively inflamed tender or swollen joints, and displayed a $<50 \%$ improvement of the tender or swollen joints, even after treatment with a maximal tolerated dose of MTX (<15 mg/week) at a stable dosage, for at least three months, without other DMARDs, and who had been receiving no more than $10 \mathrm{mg}$ of prednisone a day. The mean number of DMARDs used before the study was 2.1 .

\section{STUDY DESIGN AND TREATMENT PROTOCOL} With the consent of the patients, all were treated with a staged approach using CSA (Cypol-N, Chong Kun Dang, Seoul, Korea) for 24 weeks to induce clinical improvement, followed by HCQ for 16 weeks to maintain the improvement. The dose of CSA was initially $2.5 \mathrm{mg} / \mathrm{kg} / \mathrm{day}$ and increased to $4 \mathrm{mg} / \mathrm{kg} /$ day according to the guideline for treatment with CSA, suggested by Panayi and Tugwell. ${ }^{9}$ Combination treatment with drugs known to interact with CSA was prohibited. The following patients were removed from the trial: patients expressing a wish to discontinue the trial, 

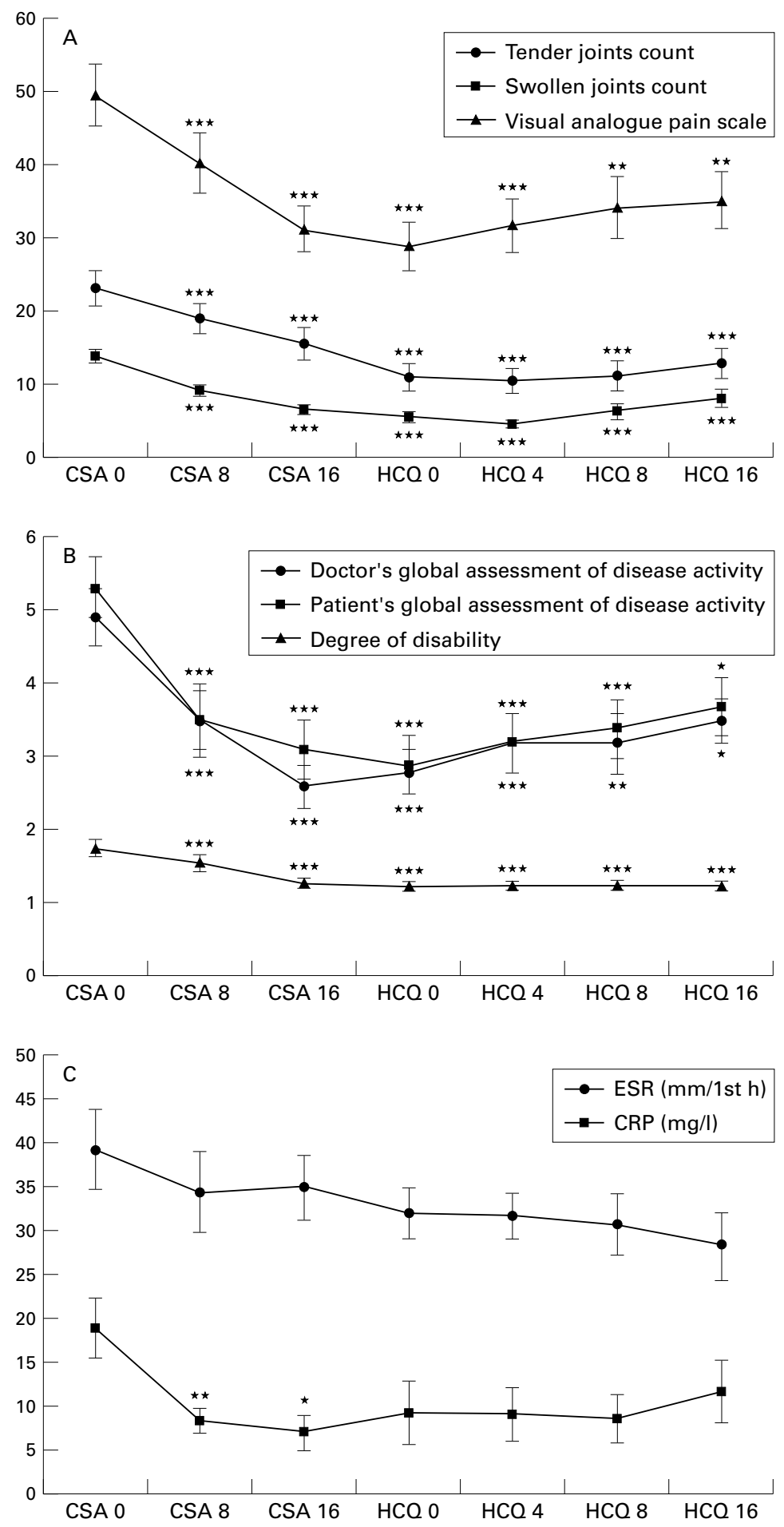

Figure 1 Outcomes of treatment with cyclosporin A (CSA) followed by a switch to hydroxychloroquine (HCQ) in patients with rheumatoid arthritis. CSA $0=$ study entry; CSA $8=$ eight weeks after CSA treatment; CSA $16=16$ weeks after CSA treatment; $H C Q \quad O=24$ weeks after $C S A$ treatment; $H C Q 4=$ four weeks after switching to $H C Q$; $H C Q 8=$ eight weeks after switching to $H C Q ; H C Q 16=16$ weeks after switching to $H C Q$. Data are means (SEM) at each week. ${ }^{\star} p<0.05,{ }^{\star *} p<0.01$, and ${ }^{\star}{ }^{*} p<0.001$ denote the significance of the differences in the ACR core set measures at the entry to the trial compared with during CSA or HCQ treatment.

patients with a white blood cell level below $3 \times 10^{9} / 1$ or platelet count below $100 \times 10^{9} / 1$, patients whose creatinine and potassium levels did not improve even after the dose was decreased, and patients not adhering to the planned prescription. After 24 weeks of CSA treatment, CSA was abruptly stopped and then replaced by HCQ $400 \mathrm{mg}$. Throughout the trial, other drugs including MTX were kept at a constant dose.

CLINICAL AND LABORATORY ASSESSMENTS

Seven clinical variables (American College of Rheumatology (ACR) core set measures) were evaluated every eight weeks (or four weeks) by two rheumatologists and two nurses who were unaware of the patients' drug status, as suggested by the $\mathrm{ACR}^{10}$ : the tender joints score (TJS, an assessment of 68 joints, classified as either tender or non-tender); the swollen joints score (SJS, an assessment of 66 joints, classified as either swollen or not swollen); pain as recorded on a $100 \mathrm{~mm}$ visual analogue pain scale (VAPS); the doctor's global assessment of disease activity $(10 \mathrm{~cm}$ visual analogue scale, where $1=$ very good and $10=$ very poor); the patient's global assessment of disease activity; the degree of disability, as measured by the Health Assessment Questionnaire (HAQ); erythrocyte sedimentation rate (ESR) and C reactive protein (CRP). The clinical improvement was defined using the ACR 20\% improvement criteria. ${ }^{11}$ Relapse after a switch to HCQ was defined by $20 \%$ or more increase in all variables of TJC, SJC, doctor's and patient's global assessment of disease, as compared with the end of treatment with CSA. A safety assessment, including vital sign, a complete blood count, urine analysis, and serum determinations of liver enzyme, urea nitrogen, creatinine, and potassium was obtained at the start of the study and every eight (four) weeks. An ophthalmological examination was performed at the beginning and end of CSA or HCQ treatment.

STATISTICAL ANALYSIS

General linear mixed models with a restricted maximum likelihood estimation were used to assess the effect of CSA or HCQ on seven ACR core set measures. This type of analysis allows incorporation of incomplete data into the models. Further exploration of the differences in outcome measures of baseline and at each week was done by the Bonferroni post hoc procedure. A probability level of $<0.05$ was adopted as significant. Quantitative data were reported as means (SEM).

\section{Results}

After screening more than 60 patients, 34 patients fulfilled our inclusion criteria and participated in this trial. The mean age of the patients ( 3 male, 31 female) was 42.6 years (range 21-58). The mean disease duration was 8.7 years (range 2.5-18) and 26/34 (76\%) patients had a positive rheumatoid factor. The mean doses of prednisone and MTX throughout the trial were $5 \mathrm{mg}$ /day (range 2.5-10) and $10.6 \mathrm{mg} /$ week (range 7.5-15), respectively. All the patients had functional class II + III disease and erosions on hand $x$ ray examination were detected in 33/34 (97\%) patients.

Four patients discontinued the trial during CSA treatment - two because of gastrointestinal toxic effects at four weeks, one owing to a lack of efficacy at eight weeks, and one due to 
Table 1 Side effects associated with cyclosporin A in 32 patients available for the safety evaluation

\begin{tabular}{ll}
\hline Adverse events & Number (\%) \\
\hline Hypertrichosis & $8(25)$ \\
Gatrointestinal trouble & $6(19)$ \\
Hypertension & $5(16)$ \\
Respiratory infection & $4(13)$ \\
Paraesthesia & $3(9)$ \\
Headache & $2(6)$ \\
Increased serum creatinine ( $\geqslant 30 \%$ from baseline) & $2(6)$ \\
Oedema & $2(6)$ \\
Liver function abnormality & $1(3)$ \\
\hline
\end{tabular}

leucopenia caused by MTX at 16 weeks. Three patients refused to continue the HCQ trial immediately after the CSA treatment. Consequently, 27/34 patients completed the trial. The data of 32 patients were analysed, which accords with the intention to treat analysis. When the seven ACR core set measures were compared between the entry point and eight weeks after the start of CSA treatment, most variables were significantly reduced (TJS $\mathrm{p}<0.001$, SJS $\mathrm{p}<0.001$, VAPS $\mathrm{p}<0.001$, doctor's global assessment of disease $p<0.001$, patient's global assessment of disease $p<0.001$,
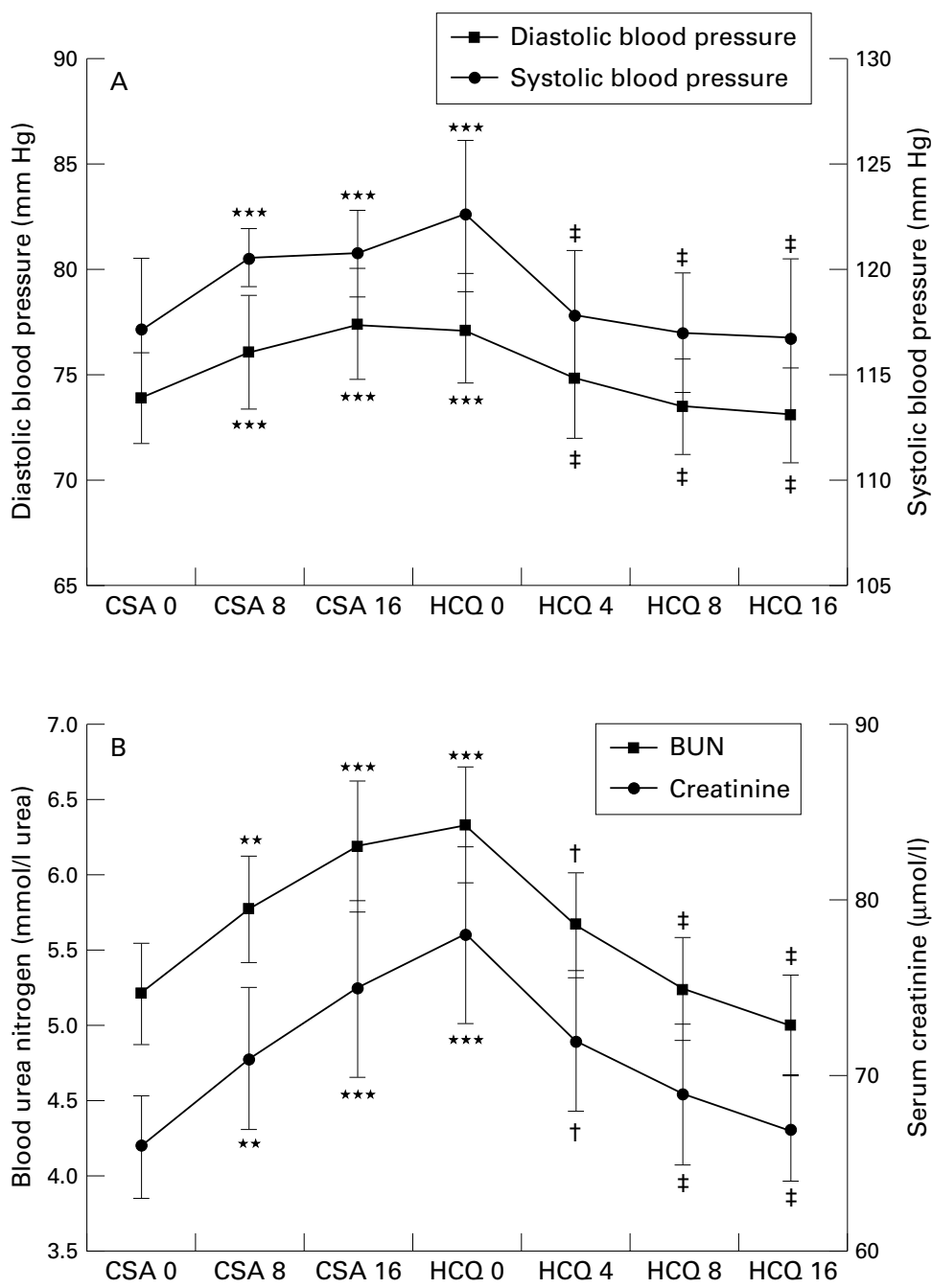

Figure 2 Changes in blood pressure, blood urea nitrogen (BUN), and serum creatinine during the treatment with cyclosporin A (CSA) followed by a switch to hydroxychloroquine (HCQ). ${ }^{\star} p<0.05,{ }^{\star} p<0.01$, and ${ }^{\star \star *} p<0.001$ denote the significance of differences in blood pressure, BUN, and serum creatinine at the entry to the trial versus during CSA or $H C Q$ treatment, while $t p<0.01$ and $\neq p<0.001$ denote the significance of differences in the above variables at $H C Q 0$ versus $H C Q 4$, 8, or 16 . See fig 1 for definitions.
HAQ $\mathrm{p}<0.001$, ESR $\mathrm{p}=\mathrm{NS}$, CRP $\mathrm{p}<0.003)$. These variables were further decreased at 16 and 24 weeks after CSA administration, and the maximal effect of CSA was achieved at 24 weeks (fig 1).

Compared with the entry into the trial, the patients switched from CSA to HCQ still displayed significantly lower levels of most variables, determined at 28,32 , and 40 weeks (significance at 40 weeks: TJS $\mathrm{p}<0.001$, SJS $\mathrm{p}<0.001$, VAPS $\mathrm{p}<0.01$, doctor's global assessment of disease $\mathrm{p}=0.034$, patients' global assessment of disease $\mathrm{p}=0.015$, HAQ $\mathrm{p}<0.001$, ESR $\mathrm{p}=\mathrm{NS}$, CRP $\mathrm{p}=\mathrm{NS}$ ). In a comparison of variables between the start and the end of HCQ treatment, most variables tended to be increased, but none of these changes were statistically significant (fig 1). According to the ACR 20\% improvement definition, ${ }^{10} 15 / 27$ (56\%) patients had improved at 24 weeks after CSA treatment. Of the 15 patients whose disease was improved by CSA, three patients $(20 \%)$ relapsed during HCQ treatment. At 16 weeks after the switch to HCQ, 12/27 patients remained improved and two newly improved cases appeared.

Thirty two patients were available for the safety evaluation. Two patients who dropped out of the trial owing to leucopenia caused by MTX or because of a lack of efficacy could not be included in the safety evaluation because they had no side effects associated with CSA until the final visit. Among 32 patients, 19 patients $(59 \%)$ had at least one side effect during CSA treatment (table 1). The mean levels of systolic and diastolic blood pressure, blood urea nitrogen, and serum creatinine were significantly raised from 8 to 24 weeks after CSA treatment $(p<0.001$ for all variables at 24 weeks) (fig 2).

After the switch to HCQ, hypertrichosis, gastrointestinal trouble, and hypertension, which had been noted during CSA treatment, completely disappeared. The side effects associated with HCQ were detected in 2/27 patients (one gastrointestinal trouble and one upper respiratory tract infection), but retinal toxicity was not found. Raised blood pressure, blood urea nitrogen, and serum creatinine associated with CSA returned to normal after switching to HCQ (fig 2).

\section{Discussion}

Despite the therapeutic advantage of CSA, its use has been reserved for patients with good renal function and severe progressive disease because of its side effects. Another concern about CSA is that if it is abruptly stopped or the dose decreased a relapse of disease activity may occur. In this study, patients who changed from CSA to HCQ still had significantly lower levels of most variables for disease activity up to 16 weeks after the switch to HCQ, despite the tendency for recurrence of disease activity. This observation supports an earlier finding that chloroquine was as effective as CSA in patients with RA with less severe disease symptoms. $^{5}$

In a trial comparing the total costs of DMARDs in RA, HCQ had the lowest 
monitoring costs for office visits, probably owing to the low toxicity. ${ }^{12}$ In this study, 19/32 $(59 \%)$ patients had at least one side effect during MTX plus CSA treatment, whereas toxicity associated with CSA completely disappeared after switching to HCQ. Therefore, from the viewpoint of a benefit-cost ratio, a switch to HCQ may be better than continuous CSA because it abrogates most of the adverse effects of CSA without a significant loss of efficacy.

This is a pilot experiment showing that treatment with CSA followed by a switch to HCQ is effective in patients with RA partially responding to MTX, and minimises the adverse effects of CSA. Because there was no control group, we cannot exclude the possibility that the results may be explained by some non-specific effect or bias. In addition, in a comparison of the efficacy of HCQ versus CSA, the lack of significance may be a statistical power problem derived from the small sample size. Further studies which include more patients with a control group and have a longer follow up of HCQ are needed.

The authors thank Sang-Jun Youn for assistance with the statistics. We are also grateful to Dr Jeong-Ah Shin for typing the manuscript.

Supported by grants from Chong Kun Dang Pharmaceutical Corporation and the Catholic University of Korea.

1 O'Dell JR, Haire CE, Erikson N, Drymalski W, Palmer W, Eckhoff PJ, et al. Treatment of rheumatoid arthritis with methotrexate alone, sulfasalazine and hydroxychloroquine, or a combination of all three medications. N Engl J Med 1996;334:1287-91.

2 Tugwell P, Pincus T, Yocum D, Stein M, Gluck O, Kraag G, et al. Combination therapy with cyclosporine and methotrexate in severe rheumatoid arthritis. N Engl J Med 1995; 333:137-41.

3 Yocum DE, Klippel JH, Wilder RL, Gerber NL, Austin HA $3 \mathrm{~d}$, Wahl SM, et al. Cyclosporin A in severe, treatmentrefractory rheumatoid arthritis. A randomized study. Ann Intern Med 1988;109:863-9.

4 Tugwell P, Bombardier C, Gent M, Bennett KJ, Bensen placebo in patients with rheumatoid arthritis. Lancet 1990; 335:1051-5.

5 Landewe RBM, Goei The HS, van Rijthoven AWAM, Breedveld FC, Dijkmans BAC. Randomized, double-blind, 24 -week controlled study of low-dose cyclosporine versus chloroquine for early rheumatoid arthritis. Arthritis Rheum 1994;37:637-43.

6 Felson DT, Anderson JJ, Meenan RF. The comparative efficacy and toxicity of second-line drugs in rheumatoid arthritis. Results of two metaanalyses. Arthritis Rheum 1990;33:1449-61.

7 Fries JF, Williams CA, Ramey D, Bloch DA. The relative toxicity of disease-modifying antirheumatic drugs. Arthritis Rheum 1993;36:297-306.

8 Arnett FC, Edworthy SM, Bloch DA, McShane DJ, Fries $\mathrm{JF}$, Cooper NS, et al. The American Rheumatism Association 1987 revised criteria for the classification of rheumatoid arthritis. Arthritis Rheum 1988;31:315-24.

9 Panayi GS, Tugwell P. The use of cyclosporin A microemulsion in rheumatoid arthritis: conclusions of an international review. Br J Rheumatol 1997;36:808-11.

10 Felson DT, Anderson JJ, Boers M, Bombardier C, Chernoff M, Fried B, et al. The American College of Rheumatology preliminary core set of disease activity measures for preliminary core set of disease activity measures for 36:729-40.

11 Felson DT, Anderson JJ, Boers M, Bombardier C, Furst D, Goldsmith C, et al. American College of Rheumatology preliminary definition of improvement in rheumatoid arthritis. Arthritis Rheum 1995;38:727-35.

12 Prashker MJ, Meenan RF. The total costs of drug therapy for rheumatoid arthritis. A model based on costs of drug, monitoring, and toxicity. Arthritis Rheum 1995;38:31825. 